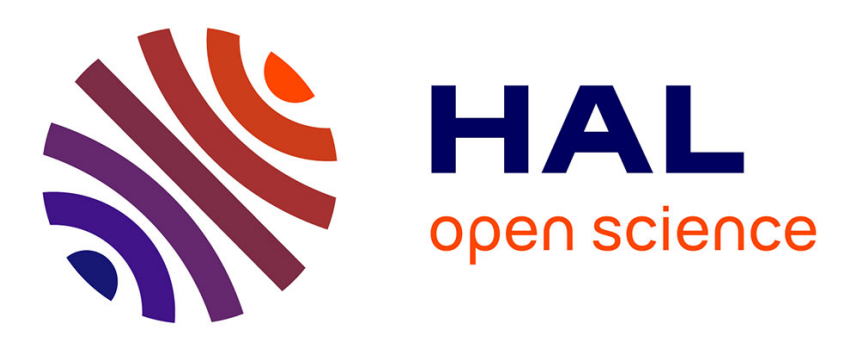

\title{
Stability polygons reshaping and morphing for smooth multi-contact transitions and force control of humanoid robots
}

\author{
Hervé Audren, Abderrahmane Kheddar, Pierre Gergondet
}

\section{To cite this version:}

Hervé Audren, Abderrahmane Kheddar, Pierre Gergondet. Stability polygons reshaping and morphing for smooth multi-contact transitions and force control of humanoid robots. Humanoids, Nov 2016, Cancun, Mexico. pp.1037-1044， 10.1109/HUMANOIDS.2016.7803399 . lirmm-01256512v2

\section{HAL Id: lirmm-01256512 https://hal-lirmm.ccsd.cnrs.fr/lirmm-01256512v2}

Submitted on 12 Oct 2016

HAL is a multi-disciplinary open access archive for the deposit and dissemination of scientific research documents, whether they are published or not. The documents may come from teaching and research institutions in France or abroad, or from public or private research centers.
L'archive ouverte pluridisciplinaire HAL, est destinée au dépôt et à la diffusion de documents scientifiques de niveau recherche, publiés ou non, émanant des établissements d'enseignement et de recherche français ou étrangers, des laboratoires publics ou privés. 


\title{
Stability polygons reshaping and morphing for smooth multi-contact transitions and force control of humanoid robots
}

\author{
Hervé Audren $^{1}$, Abderrahmane Kheddar ${ }^{1,2}$ and Pierre Gergondet ${ }^{1}$
}

\begin{abstract}
In this paper we provide hindsight on how to use the information provided by the explicit computation of the stability polygon and build on task-based QP controllers to achieve both stable multi-contact smooth transitions and force control without jeopardizing stability. This entails computing stability polygons with unilateral and bilateral contacts and devising a method to continuously constrain the CoM position throughout multi-contact motion in order to effectively regulate the forces applied on the environment while avoiding hard constraints on the CoM and discontinuity between stances.

Index Terms - Body balancing, Locomotion, Humanoid Dynamics
\end{abstract}

\section{INTRODUCTION}

Multi-contact technology [1], [2] can be used in several tasks such as climbing stairs using handrails or egress/ingress a car, or more generally access confined spaces. While several works have proposed efficient regulation control strategies in multi-contact configurations [3]-[6], transitions between contact stances is still to be researched ${ }^{1}$.

One can enforce stability by controlling the Center of Pressure (CoP) at each contact to remain within the convex hull of its area [5]-[9]. This criterion can be added to the QP controller as a constraint. Yet, in these implementations, the CoM was given as a high-level task, and the CoP or contact forces were regulated often in the null-space or in conjunction with the CoM task. Moreover, CoP are illconditioned upon contact removal because they only exist when the normal contact force is non-zero. All papers using CoP treat this issue with an ad-hoc counter-measure.

In order to smoothly transition from contacts, we want to specify only a desired force profile on a given contact without endangering stability. Whole body controllers typically solve an optimization problem that aims at finding compatible joint accelerations, $\ddot{q}$ and contact forces $\lambda$ minimizing a set of task errors under constraints. Stability objectives typically only depend on the current joint state (position, velocity and acceleration) $q, \dot{q}, \ddot{q}$. Thus, adding a task to minimize the 2 norm of $\lambda$ will not result in modification of angular values: the best local solution is redistributing the internal torques to fall as little as possible.

One usual way to circumvent the local-view issue is to use a preview controller to enhance the motion with a flavor of dynamics [8], [10], but slow multi-contact motion can be

\footnotetext{
* This work was supported by the H2020 European Project COMANOID

1 CNRS-AIST Joint Robotics Laboratory UMI3218/RL, Tsukuba, Japan

2 CNRS-UM LIRMM, Interactive Digital Human, Montpellier, France

${ }^{1}$ Among the conclusions of the panelists of IEEE-RAS Humanoids 2015's workshop on Whole-Body Multi-Task Multi-Contact Humanoid Control
}

achieved without preview [2], using only a closed-loop local task-based controller.

To ensure that the desired force is indefinitely sustainable, we want the controller to move the CoM to a stable region. However, this approach is not directly applicable as the CoM position only depends on $q$ : a task error $v(q, \lambda)$ cannot be turned into a quadratic objective $\mathcal{T}$ that depends only on $\ddot{q}$ and $\lambda$. Indeed, to do so one must differentiate $v$ with respect to time, but $\lambda$ is our optimization variable.

Forcefully setting the CoM to a precise location will require to set gains that will interfere with almost all the other tasks, such as end-effectors trajectories. This is generally a very conservative measure. Thus, we may want the CoM objective to be among the tasks to be achieved at best when this is needed, but it should be allowed to freely move in a known region of stability, that can be a hard constraint.

In this study, we will limit our developments to multicontact with quasi-static motions. Hence, constraining the CoM to remain within the frictional static stability polygon is an acceptable criterion (ZMP assumes infinite friction at contact). The static stability is a polygon and allow us to have a better comprehension before moving to the general $3 \mathrm{D}$ case in future work. Yet this shape is neither modified according to desired contact forces nor continuous across contact changes. For the latter point, including it directly as a constraint in our QP controller would lead to its immediate violation upon contact transition.

To deal with the latter drawbacks, our main contribution is to propose a novel continuation method based on optimal matching of points to interpolate between convex polygons (those resulting from multi-contact stability region computation). This allows us to smoothly reshape the region of stability during contact removal and addition and hence make QP controllers robust to contact changes.

By formulating a pure CoM based constraint, we effectively decouple the CoM placement from the force regulation problem which allows us to perform force control in multicontact. Indeed, we can deform the static stability polygon obtained from the geometrical and frictional information of a stance to take into account the desired contact forces. Then, using a simple impedance control scheme, we regulate the applied force to the objective one without endangering stability. This is another novel contribution, as we do not move the CoM to a predetermined location, but inside a formally defined polygon that guarantees stability with respect to a certain force limitation. We note that our work applies to torque and position controlled robots, but it has more impact and value for the latter ones as redistributing 
internal torques has no effect on them.

We recall in section II the static stability polygon computation, then how we included it as a constraint our controller in section IV. We introduce our idea to smoothly constrain the CoM in section III before applying it to the stairs case in section V. We present a way to efficiently combine tasks with this constraint in section VI and present results obtained in another multi-contact scenario in section VII.

\section{COMPUTATION OF THE STABILITY POLYGON}

We use the algorithm in [11] to compute the stability polygon for static postures provided by the multi-contact planner. The exact stability polygon lies between an inner and an outer approximation, that are built iteratively from solving a series of second-order cone programs of the form:

$$
\begin{array}{cl}
\underset{\max }{\operatorname{mam}} & d^{T} \widetilde{c o m} \\
\text { s.t. } & A_{1} x+A_{2} \widetilde{c o m}=t \\
& \|B x\| \leq u^{T} x
\end{array}
$$

The solution $\widetilde{\mathrm{com}}^{*}$ of this problem is an extremal stable CoM position in the direction $d$ i.e. there exists a set of forces $x$ realizing $t=\left[\begin{array}{ll}m g & 0\end{array}\right]^{T}$ under the non-linearized friction cones constraints defined by $B$ and $u$. This wrench is composed of the total wrench generated by a set of forces $x$, at the origin, computed using the transformation matrix $A_{1}$ and the gravity wrench computed with $A_{2}$ given a CoM position $\widetilde{\mathrm{com}}$. For each of these problems, $\widetilde{\mathrm{com}}^{*}$ is added to the inner approximation, while the half-plane defined by $\left\{\widetilde{\text { com }} \in \mathbb{R}^{2} \mid d^{T} \widetilde{\text { com }}>d^{T} \widetilde{\text { com }}^{*}\right\}$ is added to the outer approximation. At each step, the difference between these two approximations is a set of triangles. The search direction for the next step is perpendicular to the edge of the inner approximation forming the triangle of maximum area. The algorithm stops when the difference between the outer and inner approximations area is less than a given precision $\sigma$. The authors prove that there is a strict upper bound on the number of iterations needed to reach $\sigma$.

In its original expression, this method is not applicable to the case of mixed unilateral-and-bilateral contacts, that we are using in our experiments [2]. The support region may be unlimited in some directions. Thus, we need additional constraints limiting the search region. As our model of bilateral contacts is simply a set of points with non-aligned friction cones, we still can use Equation 1 providing additional constraints on the acceptable CoM positions. Accordingly, we can either use a constraint $|\widetilde{c o m}|<r$ that will constrain the CoM to a rectangular (polygonal) region or a conic constraint of the form $\|\widetilde{c o m}\|<r$ that will constraint the CoM to a circle. A circular constraint is difficult to approximate by a polygon. However, it may be closer to the real geometric constraint: using a maximum limb length per contact, we can write a series of constraints $\|\widetilde{c o m}-l\|<r$ that ensure that the CoM never goes further than $d$ from the contact at position $l$.

The stability region is unlimited is some directions because we can apply arbitrary forces on opposite contact points, allowing us to compensate for any momentum. In fact, the robot cannot apply infinite torques. Translating the torque constraint into a precise force limit of the form $|x|<f_{m}$ requires setting $f_{m}$ from the robot posture, whereas we reason on the CoM model to compute the stability polygon. Instead, we use a reduced torque constraint, $|T x|<\tau_{m}$ where $T$ represents the cross product between the contact points and the contacting link. In this case, $\tau_{m}$ can be obtained from the characteristics of the link that is in contact (e.g. gripper actuators or feet/ankle actuators). This constraint is linear because we consider non-sliding contacts.

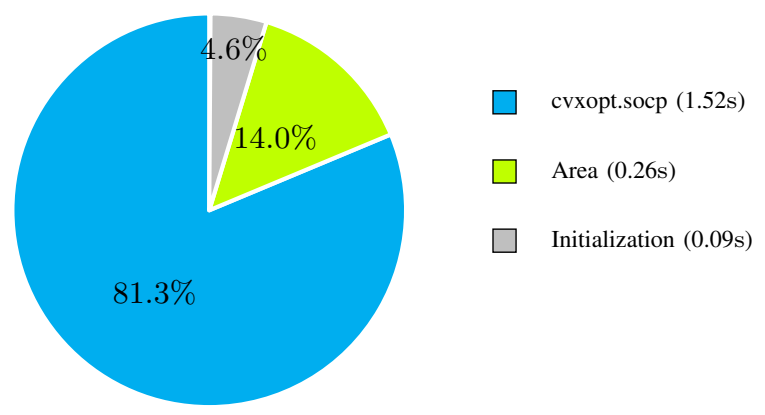

Fig. 1: Pie chart showing as angular sections the repartition of computation time for 150 iterations: total time $1.87 \mathrm{~s}$

This method is quite fast but not enough for real-time. It takes few seconds for over a hundred iterations. To solve the second-order cone programs, Equation 1, we use the convex optimisation package CVXOPT [12] that natively supports conic constraints. Our implementation is sub-optimal as we recompute the area spanned by every edge at every iteration but the cost for solving each problem is by far the most consuming task. This means that even if we curb the time spent finding the next best edge, the overall runtime of the algorithm will not be significantly improved.

\section{MORPHING STABILITY POLYGONS}

Now, we are able to compute the stability region for each contact configuration. It is a polygon that can be reshaped in a more conservative form when needed. But more importantly, the static stability can be integrated in our controller as a hard constraint through 'CoM in Polygon' equations. When the robot changes contact configuration, the stability polygon shape for the following configuration is different from the current one. We do not want to reset the problem or to design a specific CoM task. Instead, we would like the constraints to continuously deform from a stability shape to the other while subscribing the CoM all along the deformation. In this case, the transition from a contact configuration to another is made smoothly while keeping hard constraints on the stability.

If we do not enforce the CoM to be maintained within a moving/morphing polygon, the QP controller might often start from an infeasible constraint set and fails. Controller failure is solved by our new Algorithm 1 to smoothly morph the stability polygons between stances and this is done within the QP controller. 


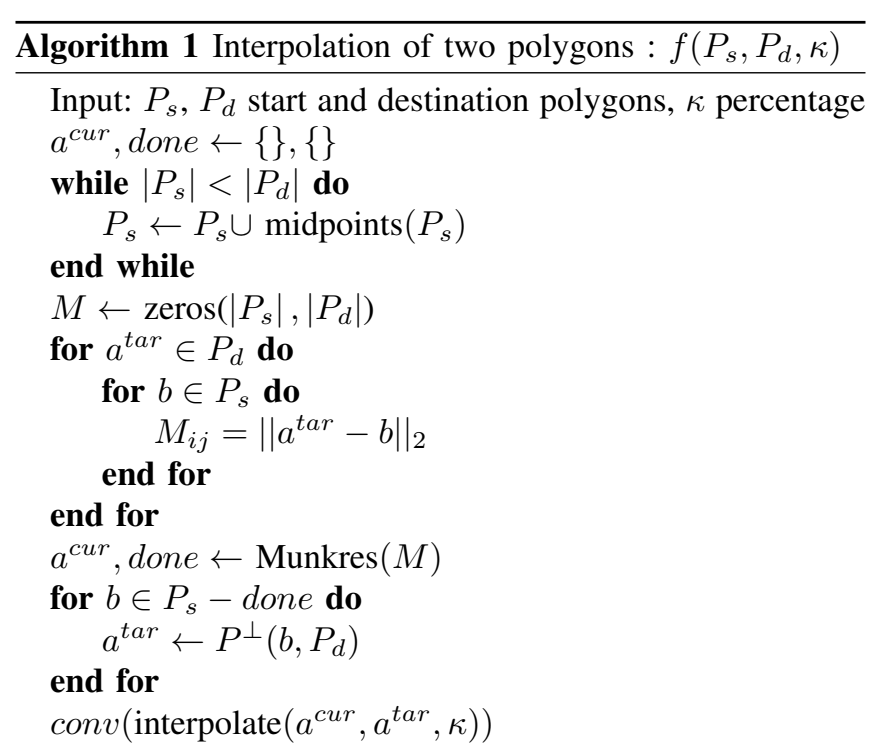

It is not necessary to use state-of-the-art 2D or 3D shape morphing found in the computer graphics and animation community, see e.g. [13], [14] because in this study we only morph 2D convex polygons. Furthermore, as we are interested in adding and removing contacts one at a time, successive stability polygons have a non-empty intersection. In particular, when adding a contact, the previous stability polygon is entirely included in the new one. Thus, our successive polygons will be both convex and have non empty intersections, which allows us to use a simpler, faster, morphing algorithm. Morphing polygons is typically done in three steps:

- Adding points to the shapes;

- Finding a correspondence between the points from the start and target shape;

- Generating intermediate shapes by interpolating each of these couples.

The main difficulty when morphing polygons is that if corresponding points are badly chosen, the resulting polygon will self-intersect during interpolation.

To solve the problem of self-intersection during morphing, we use the Munkres (Hungarian) algorithm [15]. This algorithm solves the assignment problem, i.e. finds a minimum weight matching in a weighted bipartite graph. In our case, we want to match every point from the target polygon to one from the current polygon while minimizing the sum of distances between each couple of matched points. In order to warrant that the current polygon has more points than the target polygon, we first extend the current polygon with the midpoint of each edge until it contains more points than the target. The result of the Munkres algorithm is the minimal, in terms of distance travelled, set of points $a^{\text {cur }}$ from the current polygon matching all points $a^{t a r}$ from the target polygon. The remaining $b$ points of the current polygon are mapped to their orthogonal projections onto the target polygon. The intermediate shape is generated by interpolating each couple of points by a percentage $\kappa$. Figure 2 presents the results obtained on simple shapes.

The Munkres algorithm is in $\mathcal{O}\left(n^{3}\right)$, yet pre-computations are possible. The start and target polygons only depend on the geometry and friction properties of each stance. Then, although the algorithm presented here directly gives back the interpolation, it is much more interesting to save the result, that is the set of correspondences $a^{\text {cur }} \rightarrow a^{\text {tar }}$ and then only compute the interpolation between each couple of points for a given morphing percentage $\kappa$ at each time-step. Moreover, as we usually use a few tens of points, running the Munkres algorithm is not very costly. To map a 25 -sided polygon onto a 50 -sided one, it takes about $0.67 \mathrm{~s}$. Solving the assignment problem takes about $95 \%$ of the time.

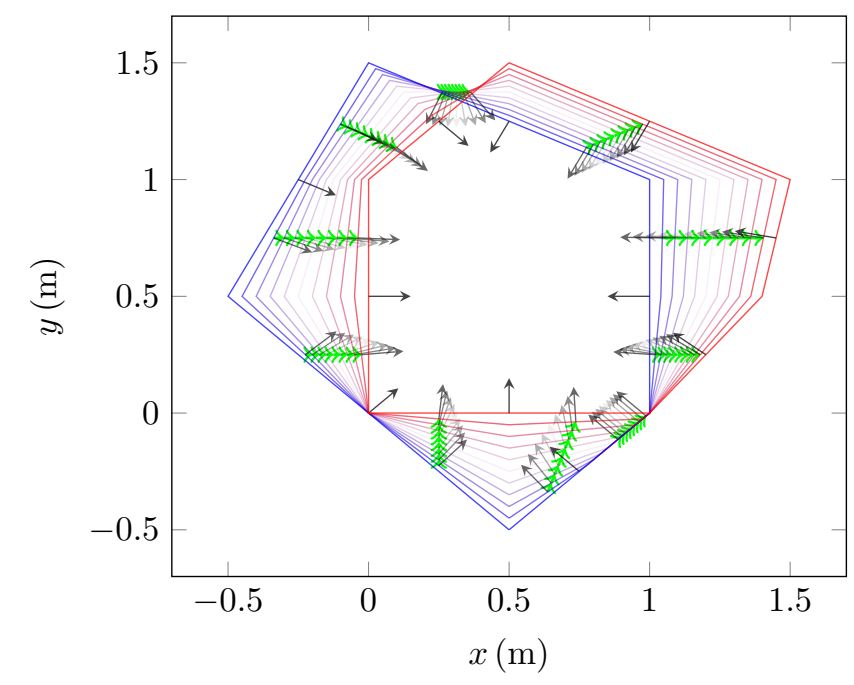

Fig. 2: Stability 2D polygon morphing: $P_{s}$ in blue, $P_{d}$ in red, the green and black arrows show the motion and the normals directions of the edges respectively.

\section{TASK-BASED CONTROLLER}

Now we discuss how this morphing is integrated in the controller. We control our humanoid robot using the multicontact QP task controller described in [2], which compact form is:

$$
\begin{array}{ll}
\min _{y} & \frac{1}{2} y^{T} Q y+c^{T} y \stackrel{\text { def }}{=} \sum_{i} \beta_{i} \mathcal{T}_{i}(y) \\
\text { s.t. } & A y \leq B ; C y=D
\end{array}
$$

where $y$ is the decision variable representing both the joint accelerations $\ddot{q}$, and the intensity of the forces $\lambda$ applied at each contact point. The problem is written as a sum of quadratic or linear objectives weighted by $\beta_{i}$ (task gains). Encoded constraints include joint position, velocity and torque limits, non-sliding contacts, and non-desired collisions avoidance. Typical tasks are written either directly or using the set-point objective formulation and comprise a wide variety of objectives [2]. Here, we focus on the CoM task.

To subscribe the CoM within a moving polygon, we use a collision constraint as in [2], [16]. However, in our case 
the CoM interacts with a changing shape (see next section), which means that we have to take into account the speed and acceleration of the edges w.r.t the CoM, that is:

$$
\begin{aligned}
& \dot{\delta}=n^{T} \cdot(J \dot{q}-\dot{p}) \\
& \ddot{\delta}=\dot{n}^{T} \cdot(J \dot{q}-\dot{p})+n^{T} \cdot(\dot{J} \dot{q}+J \ddot{q}-\ddot{p})
\end{aligned}
$$

where $p, n$ are respectively the position and normal of the CoM projection on one such edge, $\delta$ the resulting distance, and $J$ the CoM Jacobian. We formulate a constraint:

$$
\begin{gathered}
u=J \dot{q}-\dot{p} \quad e=\dot{J} \dot{q}-\ddot{p} \\
-\mathrm{d} t n^{T} J \ddot{q} \leq \xi \frac{\delta-\delta_{s}}{\delta_{i}-\delta_{s}}+\dot{\delta}+\mathrm{d} t\left[\dot{n}^{T} u+n^{T} e\right]
\end{gathered}
$$

with $\xi$ a damping coefficient, $\delta_{i}$ and $\delta_{s}$ the interaction and safety distances respectively. Activating the above constraint whenever $\delta<\delta_{i}$ will ensure that $\delta$ is never smaller than $\delta_{s}$.

In a number of cases, the quantities $\dot{p}, \ddot{p}, n, \dot{n}$ can be explicitly computed from the mapping obtained by applying Algorithm 1. Indeed for every ordered vertex $v_{k}$ of our current (convex) polygon that is the interpolate between $a_{k}^{c u r}$ and $a_{k}^{t a r}$ at $\kappa(t), t$ being the time:

$$
\begin{array}{ll}
v_{k}(\kappa) & =a_{k}^{\text {cur }}(1-\kappa)+a_{k}^{\text {tar }} \kappa \\
\frac{\partial^{n} v_{k}}{\partial t^{n}} & =\left(a_{k}^{\text {tar }}-a_{k}^{\text {cur }}\right) \frac{\partial^{n} \kappa}{\partial t^{n}} \quad \forall n \geq 1
\end{array}
$$

Thus, for every point $p_{k}$ of the segment $\left[v_{k}, v_{k+1}\right]$ at a distance $\alpha_{k}$ from the $v_{k}$ and the associated normal $n_{k}$ :

$$
\begin{aligned}
\frac{\partial^{n} p_{k}}{\partial t^{n}} & =\sum_{i=0}^{n}\left(\begin{array}{c}
n \\
i
\end{array}\right) \frac{\partial^{i} \alpha_{k}}{\partial t^{i}} \frac{\partial^{n-i} v_{k}}{\partial t^{n-i}}+\frac{\partial^{i}\left(1-\alpha_{k}\right)}{\partial t^{i}} \frac{\partial^{n-i} v_{k+1}}{\partial t^{n-i}} \\
n_{k} & =\left[\begin{array}{cc}
0 & -1 \\
1 & 0
\end{array}\right]\left(v_{k+1}-v_{k}\right) \stackrel{\text { def }}{=} N\left(v_{k+1}-v_{k}\right) \\
& =N\left[a_{k+1}^{\text {cur }}(1-\kappa)+a_{k+1}^{\text {tar }} \kappa-\left(a_{k}^{\text {cur }}(1-\kappa)+a_{k}^{\text {tar }} \kappa\right)\right] \\
& =(1-\kappa) N\left(a_{k+1}^{\text {cur }}-a_{k}^{\text {cur }}\right)+\kappa N\left(a_{k+1}^{\text {tar }}-a_{k}^{\text {tar }}\right) \\
& =(1-\kappa) n_{a_{k}^{\text {cur }}}+\kappa n_{a_{k}^{\text {tar }}} \\
\frac{\partial^{n} n_{k}}{\partial t^{n}} & =\left(n_{a_{k}^{\text {cur }}}-n_{a_{k}^{\text {tar }}}\right) \frac{\partial^{n} \kappa}{\partial t^{n}}
\end{aligned}
$$

Whenever two points of the current polygon map to the same point of the destination polygon, i.e. $n_{a_{k}^{t a r}}=\left[\begin{array}{ll}0 & 0\end{array}\right]$, the direction of $n_{k}$ is constant but its norm is strictly decreasing. To avoid numerical issues whenever $\left\|n_{k}\right\|$ is small, we consider $n_{k}$ constant with respect to $\kappa$ in this case.

Thus, knowing the $n^{\text {th }}$ time derivative of $\kappa$ and $\alpha$, one can compute the $n^{\text {th }}$ time derivative of the segments' position and their normals. However, when $\kappa$ or $\alpha$ depend implicitly on the time, these quantities have to be evaluated through numerical differentiation. We can make the following approximations to neglect high-order terms:

- As the CoM only interacts with edges closer than $\delta_{i}$, it usually interacts with few edges at a time. Because all points are interpolated uniformly and because the constraint pushes the CoM perpendicularly to the edge, variations of $\alpha_{k}$ are small during interaction: we consider that $\frac{\partial^{n} \alpha_{k}}{\partial t^{n}}=0 \forall n>0$.
- In most of our use-cases, we interact when the interpolation is almost done (the CoM is generally nearby the barycenter of the polygons. At this moment, $\ddot{p}$ is either zero (when we interpolate at constant speed) or negative (using quadratic interpolation, the polygon is contracting and slowing down): we set $\ddot{p}=0$. This puts a harder bound on the constraint.

Overall rotation speeds are usually small compared to the homothetic part of the transformation, but we can not neglect $\dot{n}$. Depending on the shape of the support polygons, local rotations can be important, such as in the top corner of the polygon in Figure 2.

\section{Limiting THE COM REGION DURING MOTION}

For the sake of clarity and without loss of generality, we illustrate our approach using the stair climbing with handrail task by our humanoid robot (HRP-2Kai). In our experiment trials the robot applied too much forces on the handrail. Although part of the excess of force is due to positioning error and would be solved by applying lowerlevel compliance, part of it comes from the fact that our QP controller does not explicitly reduce the force applied on the gripper. Adding a task of the form min $\left\|S_{\text {gripper }} \lambda\right\|^{2}$ did minimize the force and torque computed, but kept the posture unchanged meaning that the force applied by the real robot would remain high (this was also observed for a torque controlled robot in [5], p. 288, last sentence). Thus, we want to modify the trajectory such that the force applied on the gripper dimishes without endangering stability.

\section{A. Linking force limitation and interpolation}

From intuition, maintaining the CoM in a shape that is similar to the static stability polygon of a restricted set of contacts should reduce the force applied on the others. We can compute the real static stability polygon with an additional force constraint: for a set of real numbers $\gamma \in$ $[0,1]$ we compute the static stability polygon as described in section II with an additional constraint on the forces applied on each contact point of the grippers:

$$
\left\|f_{i}\right\| \leq \gamma m g
$$

Then we compute two stability polygons at each step of the climbing, i.e. every time the active set of contacts is changed: one using all contacts, the other using all contacts but those between grippers and rail, and their interpolation. We then find by dichotomy which $\kappa$ yields the the interpolation of the "full" and "restricted" polygon with the closest area to the real constrained static stability polygon. Captions of this process are shown in Figure 3 while a numerical comparison of values of $\gamma$ and $\kappa$ are shown in Figure 4.

This shows us that interpolation is a good approximation of the force constraint with restrictions:

- Because we limit the search region, the interpolation retains the shape of this limit contrarily to the constrained static stability polygon.

- $\kappa$ is a non-linear function of $\gamma$. Indeed, as a consequence of the above point, there is a "dead-zone" when the force constraint results in a shape modification smaller 


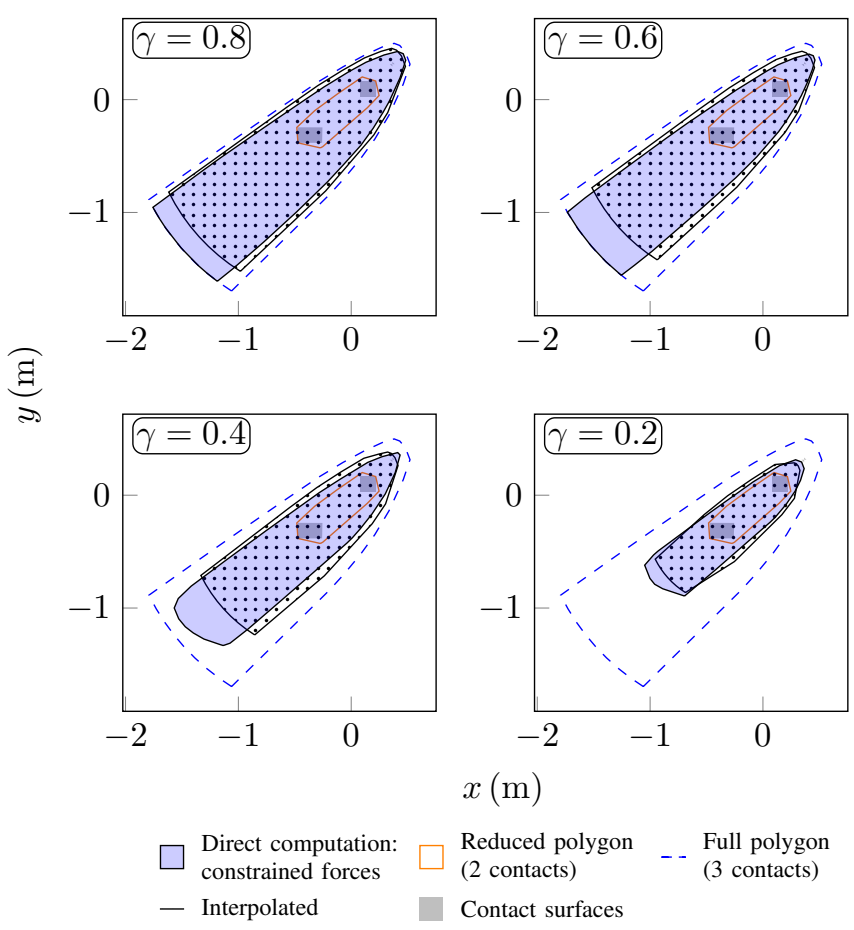

Fig. 3: Comparison between direct computation of the constrained static stability polygon and interpolation.

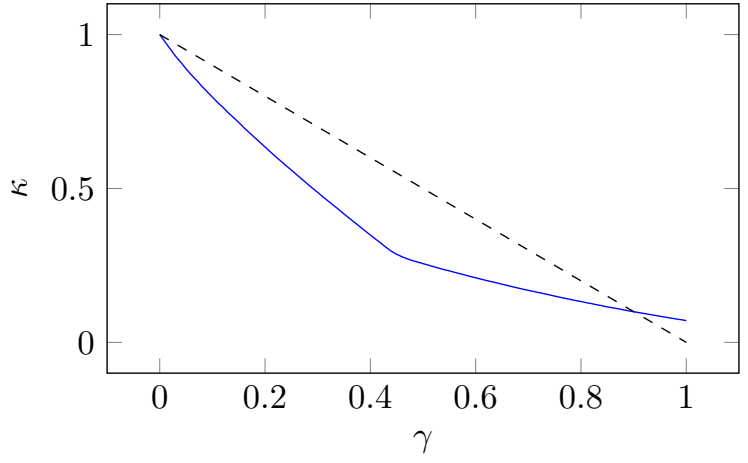

Fig. 4: Interpolation coefficient as a function of the force constraint coefficient for a 3-contact scenario. Dashed line represents $\kappa=1-\gamma$.

than the distance constraint. However, this function does not have a general expression. Indeed, even when linearizing the friction constraints, the two-dimensional stability polygon is the result of a series of nonlinear transformations. As our problem is defined as a series of inequalities describing contact geometry and friction and equalities describing stability conditions, the first step is to enumerate the vertices of the convex polyhedron hence described in the manifold of forces and $\mathrm{CoM}$ positions. This operation is called double description [17]. Then, adding a linear force limitation constraint corresponds to capping this $\mathrm{n}$-dimensional polyhedron, which may modify any number of edges, and will present discontinuities depending on which edges intersect with the constraint. Note that the two above operations can be computed in a single pass of double description, but capping would allow for efficient testing of various force limitations. Finally, the polygon is the convex hull of the projections of those vertices in the plane. Another non-linear, complex operation. It is thus impossible to derive a general relationship between $\gamma$ and $\kappa$ that is independent of the contact configuration. Moreover, as double description is an NPhard problem [18], enumerating all vertices in a highdimensional space may be much more computationally costly than repeatedly computing only the projection, as the worst-case complexity is doubly exponential. Similarly, capping in high-dimensional spaces is much more expensive than in two or three dimensional space [19].

- Interpolation is more accurate for extreme values of $\gamma$ which is a direct consequence of using interpolation.

Thus, maintaining the $\mathrm{CoM}$ inside a polygonal region using the constraint presented in section IV corresponds to limiting the force applied on the gripper. This polygonal region is smoothly interpolated from the current static stability polygon, to an intermediate one. We can still maintain a task of the CoM in the cost function with low gains and limit the CoM motion in the constraint part of the QP. Note that we can use a static stability polygon because the accelerations and speeds generated by our controller are small and because the safety distance $\delta_{s}$ acts as a stability margin.

\section{B. Results of stairs climbing}

To limit the stability region, we use the constraints of section II in the stability polygon computation. We strictly limit the torque applied on the gripper, and loosely the forces and CoM position using the following values:

$$
\left|f_{i}\right|=\left|S_{i} x\right| \leq 5 \mathrm{mg} ;\left|\tau_{i}\right|=\left|T_{i} S_{i} x\right| \leq 5 \mathrm{~N} \mathrm{~m} ;\|\widetilde{\operatorname{com}}\| \leq 1 \mathrm{~m}
$$

With $S_{i}$ a selection matrix of forces at the $i^{\text {th }}$ contact. The interpolation between the current and target polygon takes place over the course of one second. To avoid strong discontinuities when the constraint gets activated, we use quadratic interpolation, i.e. a trapezoidal speed profile. We also use relatively high damping, $\xi=0.1$, to further avoid discontinuity when activating the constraint. To make sure the QP does not fail, we allow a small violation of the constraint. The intermediate polygon is the interpolate at $90 \%$ : we need to choose a high $\kappa$ because we want to strongly limit the forces applied on the gripper. This $\kappa$ corresponds to an upper force of about $34 \mathrm{~N}$, which means that in the worst case the torque applied on the gripper is lower than our torque limit.

We first designed the motion, and checked that introducing the polygonal constraint in our controller does indeed modify the posture as shown in Figure 5. Moreover, introducing this constraint allowed us to check that our controller can indeed generate a trajectory without applying any force on the gripper.

We then modified the CoM objectives in each phase to ensure that the robot's flexibilities would not be too excited 


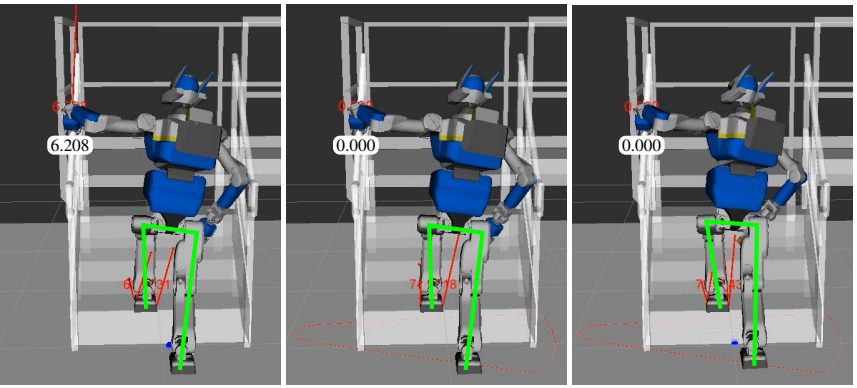

Fig. 5: Climbing the stairs with HRP-2 Kai: with neither the constraint nor gripper torque task activated (left), with only the task (middle) and with both (right)

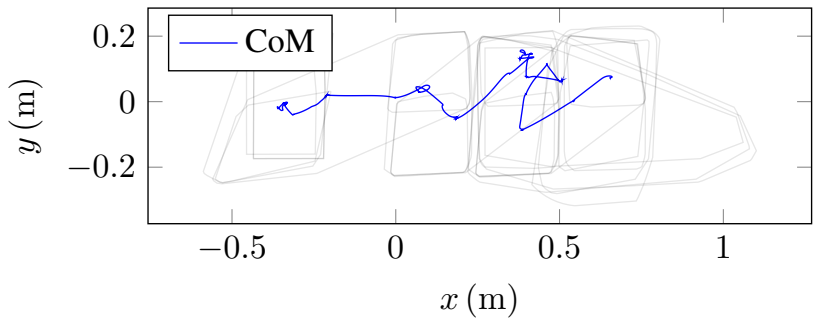

Fig. 6: CoM trajectory during experiment

during motion, and confirmed that the robot can climb the stairs in dynamic simulation.

To make sure that the robot was able to climb the stairs in the real world, it was very important to ensure that the contacts were properly established before moving on to the next phase. Thus, every time a contact has to be added, we use a $6 \mathrm{D}$ position task as a form of impedance control to regulate the contact force. Its objective $X(t)$ is updated as follows:

$$
\begin{aligned}
\delta f & =f_{m e s}-f_{o b j} \\
X(t+d t) & =X(t)+K_{p} \delta f+K_{d} \dot{\delta f}
\end{aligned}
$$

With $K_{p}, K_{d}$ positive diagonal gain matrices, $f_{m e s}$ the measured 6 dimensional wrench, $f_{o b j}$ an objective wrench. This task allows us to align the contacting bodies with the contacting surfaces by specifying a pure normal force objective as shown in the attached video.

The resulting trajectory is shown in Figure 6: while the constraint was correctly added to our QP controller, it is rarely activated. This confirms that the manual tuning concords with the stability information. Note that during this testing phase, being sure that the CoM would never exit the polygon was very useful as it allowed us to tinker without worrying about changes in other tasks disturbing the CoM. In that sense, this technique is a complement to posture generation as it adds explicit boundaries in between the known to be static stances.

However, we can see in Figure 8 that the effective force applied during the motion is often superior to the designated force. Although part of this error stems from the fact that the polygons are not recomputed according to the actual contacts taken and more generally modeling errors, most of it is due to the fact that a low-force solution only exists, but it is not the one we get when doing raw position control.

\section{COMBINING TASKS AND CONSTRAINTS FOR FORCE CONTROL}

In this section we present how to combine the CoM constraint and an impedance force control task to drive smooth removal of contacts. This is in fact a particular case of potentially more complex robot force control tasks. We also show that it is possible to recompute on the fly, but not in real-time the important polygons.

In a $n$ contact scenario, we want to force control the $i^{\text {th }}$ contact, $c_{i}$. We can then compute two geometry stability polygons:

$$
\begin{aligned}
& P_{s}=\bigsqcup\left(\left\{c_{k}\right\}_{k \in[0 \cdots n]}\right) \\
& P_{d}=\bigsqcup\left(\left\{c_{k}\right\}_{k \in[0 \cdots n] \quad k \neq i}\right)
\end{aligned}
$$

We can then interpolate between $P_{s}$ and $P_{d}$ at a rate $\kappa(t)$. We choose $\kappa(t)$ to have a trapezoidal speed profile of total duration $30 \mathrm{~s}$. Denoting $f_{k}$ the force at the $k^{\text {th }}$ contact, we can derive an objective force at that contact, $\bar{f}_{k}$ that is a function of $\kappa$. Knowing that we only control one contact, we choose:

$$
\bar{f}_{k}=m g(1-\kappa)
$$

We can then use an impedance force control task such as the one presented in section $\mathrm{V}$ to regulate the actual $f_{k}$ around $\bar{f}_{k}$ by setting $f_{o b j}$ to $\bar{f}_{k}$ at each timestep. In reality, we only modify the impedance task objective whenever $f_{k}>\bar{f}_{k}$ to only maintain $f_{k}$ under the maximum desired force.

This linear objective in $\kappa$ is in general sub-optimal as lower forces could be attained, but without an explicit relationship between $\kappa$ and $\gamma$, we have to remain conservative.

\section{ApPlicAtion to A MULti-CONTACT SETTING}

In this experiment, the controller establishes three contacts with the environment. Each of the two contacts that are added use the force control task to ensure paralellism of the contacting body and surface. When done, the robot has established three unilateral contacts with its environment: one between the right foot and the rear flat platform, one with its left foot and an inclined ramp and the last one is with its hand and an elevated flat block.

After establishing the contacts, we reposition the CoM before starting the experiment. During those few seconds, we asynchronously compute the corresponding polygons: $P_{s}$ and $P_{d}$ for every contact as well as every interpolator.

While maintaining those contacts, we interpolate $P_{s}$ towards $P_{d}$ for every contact: first for removing the right foot, then for removing the left foot and finally for removing the right hand. Another shorter experiment presented in Figure 9 and the attached video consists in doing only one interpolation, but then entirely removing the right foot.

In order to check that this technique would be applicable on the robot, we first tested it in the Choreonoid dynamics simulator [20], which is very reliable (it embeds flexibilities 

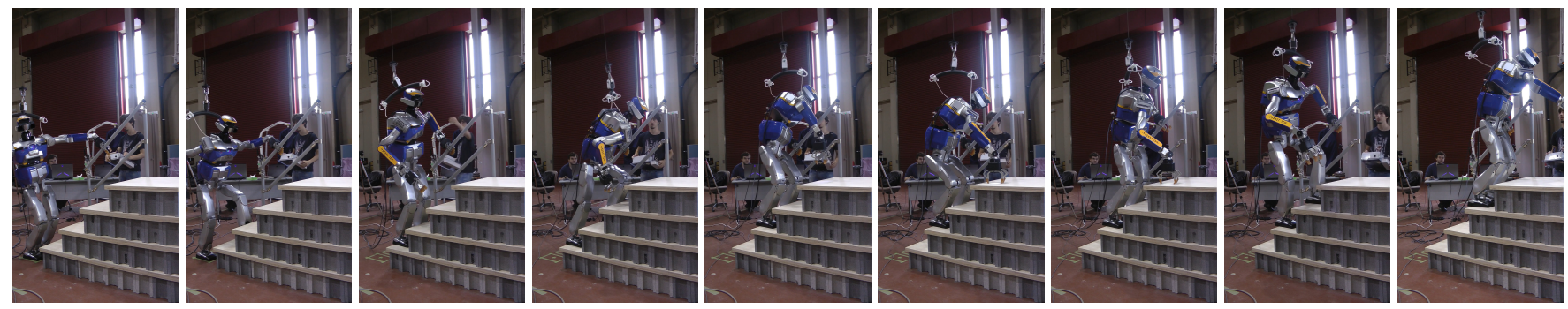

Fig. 7: Frames of HRP2 climbing the stairs using the handrail

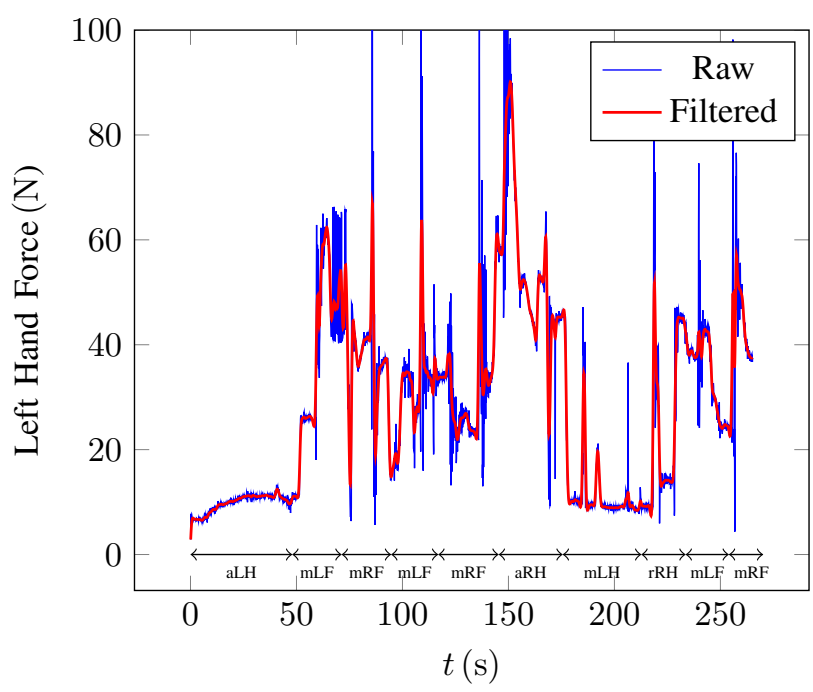

Fig. 8: Force (magnitude) applied on left hand during stairs climbing. Data was low-pass filetred by an order 2, Butterworth filter with a critical frequency of $10 \mathrm{~Hz}$. For phases, $a, m, r$ stand for "add", "move" and "remove" respectively while $R, L, F, H$ stand for "Right", "Left", "Foot", "Hand".

at the ankle and noise) to ensure that the trajectory is indeed feasible and that the robot does avoid both environment and self-collisions.

In this experiment, we do not use a CoM task, only the constraint with wide safety margin. This ensures smoothness and stability. We also add a very low weight posture task to avoid having uncontrolled joints. The last task is the impedance control task that is only inserted when interpolating for the first contact. This will allow us to confirm its importance and role. Note that without an explicit force regulation task, as the Hessian of the problem is positive definite, the controller will try to minimize $\|\lambda\|^{2}$ i.e. an equal distribution that does not reflect the real forces being applied.

Whenever we finish the interpolation (i.e. $\kappa=1$ ), the $\mathrm{CoM}$ is inside the reduced support polygon of the supporting contacts: there exists a zero-force solution -at the contact to be removed- to support the robot in this configuration. Of course, we do not know a priori if this solution fulfills the torque limits of the robot, but as we started from a feasible configuration and smoothly drove the robot to the desired one while continuously reducing the force applied on the

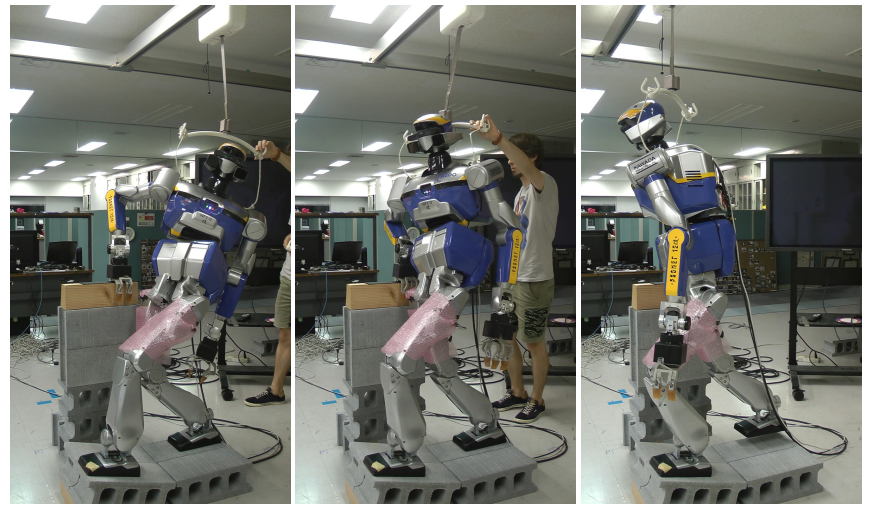

Fig. 9: Snapshots of the force control experiment with HRP-2 to smoothly remove the right foot.

contact, it is to be expected.

We show in Figure 10 how the force did decrease continuously, both in simulation and in reality albeit with slightly different profiles. We reach a zero-force configuration whenever the interpolation ends for the first contact. Note that for successive contacts, this is no longer true. When we do not explicitly aim for the desired force distribution, the robot establishes itself in a configuration that balances the contact forces with the joint torques generated by the lower level PD controller. Indeed, the worst case is shown in the third part, when interpolating to remove the hand: as the CoM is already almost inside the reduced polygon, the force applied on the left hand is only slightly reduced. However, it could safely be controlled to smoothly decrease towards zero as the interpolation index decreases as was done for the foot.

\section{CONCLUSION}

In this paper, we show that it is possible to enforce stability as a constraint in low dynamics multi-contact transitions. We devised a continuous morphing of the stability polygon as part of the constraint in QP controllers. We use this technique to limit the force applied on a given contact, whether by directly restraining the accessible region of the CoM throughout the movement or by specifying the force to be applied on a contact and displacing the CoM accordingly. Once the CoM is correctly placed, we can be sure that impedance or admittance control is usable to select the appropriate force distribution. Although we still use postures generated from planning, it is an interesting addition to it, 

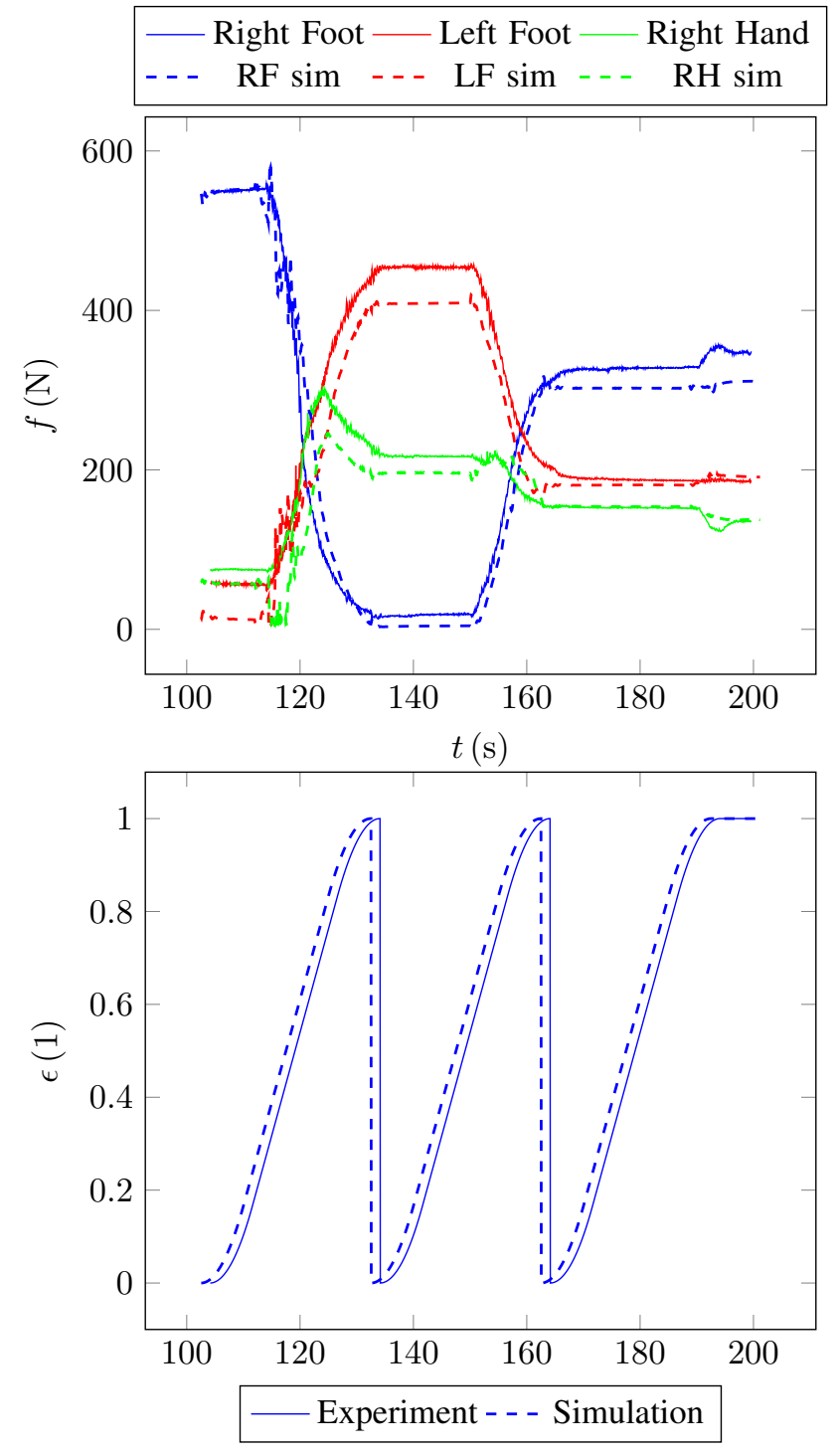

Fig. 10: Results of multi-contact experiment and simulation. Top: normal force applied on all contacts during motion. Bottom: interpolation index.

as it allows us to modify the generated motion according to our needs without any expensive re-computation. This is even more interesting as it allows us to specify that the CoM has to remain in a deformable stability region instead of explicitly forcing the CoM to remain at the planned position.

As future work, we are working on the following improvement: extending this work to dynamic motion, and closedloop control. For the former, we need to extend the theoretical part to take into account accelerations explicitly instead of relying on safety margins (this is almost done). For the latter, we need to curb the computation time of the morphing (as it will depend on online measured/estimated forces) and that of the computation of the geometric stability polygon to run with the controller on the robot embedded computer. Similarly, to account for differences between actual surface shapes and planned ones we need to efficiently compute the actual shape from the off-line planned one, possibly by using the current robot state to narrow down the search area.

\section{REFERENCES}

[1] K. Bouyarmane and A. Kheddar, "Humanoid robot locomotion and manipulation step planning," Advanced Robotics, vol. Special Issue on Cutting edge robotics in Japan, no. 26, pp. 1099-1126, July/September 2012.

[2] J. Vaillant, A. Kheddar, H. Audren, F. Keith, S. Brossette, A. Escande, K. Bouyarmane, K. Kaneko, M. Morisawa, P. Gergondet, E. Yoshida, S. Kajita, and F. Kanehiro, "Multi-contact vertical ladder climbing with an hrp-2 humanoid," Autonomous Robots, vol. 40, no. 3, pp. 561-580, 2016.

[3] C. Ott, M. A. Roa, and G. Hirzinger, "Posture and balance control for biped robots based on contact force optimization," in IEEE-RAS International Conference on Humanoid Robots, Bled, Slovenia, 2011, pp. 26-33.

[4] S.-H. Lee and A. Goswami, "A momentum-based balance controller for humanoid robots on non-level and non-stationary ground," $\mathrm{Au}$ tonomous Robots, pp. 1-16, 2012.

[5] L. Righetti, J. Buchli, M. Mistry, M. Kalakrishnan, and S. Schaal, "Optimal distribution of contact forces with inverse-dynamics control," The International Journal of Robotics Research, vol. 32, no. 3, pp 280-298, 2013.

[6] A. Herzog, L. Righetti, F. Grimminger, P. Pastor, and S. Schaal, "Balancing experiments on a torque-controlled humanoid with hierarchical inverse dynamics," in IEEE/RSJ International Conference on Intelligent Robots and Systems, Chicago, Illinois, 14-18 September 2014.

[7] L. Sentis, J. Park, and O. Khatib, "Compliant control of multicontact and center-of-mass behaviors in humanoid robots," IEEE Transactions on Robotics, 2010.

[8] H. Audren, J. Vaillant, A. Kheddar, A. Escande, K. Kaneko, and E. Yoshida, "Model preview control in multi-contact motion- application to a humanoid robot," in IEEE/RSJ International Conference on Intelligent Robots and Systems, Chicago, Illinois, 14-18 September 2014.

[9] S. Feng, E. Whitman, X. Xinjilefu, and C. G. Atkeson, "Optimization Based Full Body Control for the Atlas Robot," IEEE-RAS International Conference on Humanoid Robots, pp. 120-127, 2014.

[10] H. Dai, A. V. Valenzuela, and R. Tedrake, "Whole-body motion planning with centroidal dynamics and full kinematics," in IEEE-RAS International Conference on Humanoid Robots, Madrid, Spain, 18-20 November 2014, pp. 295-302.

[11] T. Bretl and S. Lall, "Testing static equilibrium for legged robots," IEEE Transactions on Robotics, vol. 24, no. 4, pp. 794-807, 2008.

[12] M. Andersen, J. Dahl, and V. Lieven, "CVXOPT: Python Software for Convex Optimization."

[13] T. W. Sederberg and E. Greenwood, "A physically based approach to 2-D shape blending," Proceedings of the 19th annual conference on Computer graphics and interactive techniques - SIGGRAPH '92, pp. 25-34, 1992

[14] M. Alexa, D. Cohen-Or, and D. Levin, "As-rigid-as-possible shape interpolation," Proceedings of the 27th annual conference on Computer graphics and interactive techniques - SIGGRAPH '00, pp 157-164, 2000.

[15] J. Munkres, "Algorithms for the Assignment and Transportation Problems," Journal of the Society for Industrial and Applied Mathematics, vol. 5, no. 1, pp. 32-38, 1957.

[16] F. Kanehiro, M. Morisawa, W. Suleiman, K. Kaneko, and E. Yoshida, "Integrating geometric constraints into reactive leg motion generation," IEEE/RSJ 2010 International Conference on Intelligent Robots and Systems, IROS 2010 - Conference Proceedings, pp. 4069-4076, 2010.

[17] K. Fukuda and A. Prodon, "Double Description Method Revisited," Combinatorics and Computer Science (LNCS 1120), vol. 1, pp. 91111, 1996.

[18] L. Khachiyan, E. Boros, K. Borys, K. Elbassioni, and V. Gurvich, "Generating all vertices of a polyhedron is hard," Discrete and Computational Geometry, vol. 39, no. 1-3, pp. 174-190, 2008.

[19] O. Günther and E. Wong, "A dual approach to detect polyhedral intersections in arbitrary dimensions," BIT, vol. 31 , no. 1, pp. 2-14, 1991.

[20] S. Nakaoka, "Choreonoid: Extensible virtual robot environment built on an integrated GUI framework," in 2012 IEEE/SICE International Symposium on System Integration, SII 2012, 2012, pp. 79-85. 


\section{NOMENCLATURE}

$\alpha \quad$ Distance along the segment, see equation (8), page 4

$\bar{f}_{k} \quad$ Objective force at contact $\mathrm{k}$, see equation (13), page 6

$\beta \quad$ Task weight, see equation (1), page 3

$\widetilde{c o m}$ CoM position, see equation (1), page 2

$a^{\text {cur }} \quad$ Corresponding points in $P_{s}$, see equation (1), page 2

$\delta f \quad$ Force error for compliance task, see equation (11), page 6

$\delta \quad$ Distance to a line, see equation (3), page 4

$\kappa \quad$ Morphing percentage, see equation (1), page 2

$\sigma \quad$ Error threshold to stop the algorithm, see equation (1), page 2

$a^{\text {tar }} \quad$ Points in $P_{d}$, see equation (1), page 2

$\xi \quad$ Damping coefficient, see equation (4), page 4

$A, C / B, D$ Constraint matrices/vectors, see equation (1), page 3

$A_{1}, A_{2}$ Matrices projecting the wrenches, see equation (1), page 2

$b \quad$ Points in $P_{s}$, see equation (1), page 2

$B, u \quad$ Matrices describing the friction cones, see equation (1), page 2

c Gradient vector, see equation (1), page 3

$c_{k} \quad \mathrm{k}$-ith contact, see equation (13), page 6

$d \quad$ Search direction, see equation (1), page 2

$e \quad$ Normal acceleration, see equation (4), page 4 $f_{k} \quad$ Force at contact $\mathrm{k}$, see equation (13), page 6

$f_{\text {mes }}$ Measured force at the sensor, see equation (11), page 6

$f_{\text {obj }} \quad$ Compliance task objective force, see equation (11), page 6

$J \quad$ CoM jacobian, see equation (3), page 4

$K_{d} \quad$ Compliance task derivative gain matrix, see equation (11), page 6

$K_{p} \quad$ Compliance task proportional gain matrix, see equation (11), page 6

$l \quad$ Contact position, see equation (1), page 2

$N \quad$ Normal matrix, see equation (8), page 4

$n \quad$ Normal to a line, see equation (3), page 4

$p \quad$ Position of a point on the line, see equation (3), page 4

Hessian matrix, see equation (1), page 3

CoM region radius, see equation (1), page 2

Selection matrix for contact forces, see equation (9), page 5

Target acceleration, see equation (1), page 2

Relative speed, see equation (4), page 4

Vertex of the interpolate, see equation (8), page 4

$X$ Compliance task position objective, see equation (11), page 6

$x \quad$ Set of forces, see equation (1), page 2

$y \quad$ Optimisation variable, see equation (1), page 3 